\title{
ESTUDIOS DE LA SATISFACCIÓN DE SERVICIOS HOTELEROS: REFLEXIONES Y ESTUDIO ESTADÍSTICOS
}

\author{
Carlos N. Bouza-Herrera \\ Universidad de La Habana, Cuba. \\ Darbelio Agatón \\ Universidad Autónoma de Guerrero, México
}

cbouza2002@gmail.com

Material original autorizado para su primera publicación en la revista académica REDMARKA. Revista Digital de Marketing Aplicado. https://doi.org/10.17979/redma.2015.01.015.4884

Recibido: 4 Octubre 2015

Aceptado 28 Noviembre 2015

\section{Resumen}

A partir del uso de índices de satisfacción del cliente (Consumer Satisfaction Index) se han erigido diversas metodologías para evaluar el desempeño de los hoteles. El interés primal es considerar diversos atributos y servicios y como estos conforman la satisfacción de los clientes. Dada la naturaleza del problema la población bajo estudio es dinámica. De hecho lo más usual es que la recolección de información deba ser considerada como de naturaleza censal. En algunos casos esta es complementada por encuestas especialmente diseñadas. Esta situación establece que los índices no son analizables utilizando métodos estadísticos inferenciales. Solo se puede hacer uso de métodos descriptivo y 
valerse de la modelación moderna para hacer análisis coherentes. Consideramos la posibilidad de que el gerente pueda aceptar la validez de un cierto modelo superpoblacional. Se justifica como entonces es posible hacer estudios utilizando métodos como los de Componentes.

Se ilustra lo discutido al hacer un estudio de las cadenas hoteleras de Cuba con datos recolectados en el 2005.

Palabras clave: Consumidor, índices de satisfacción

\section{Abstract}

From the use of the satisfaction index of the clients (Consumer Satisfaction Index) different methodologies has been developed for evaluating the work of hotels. The main interest is considering the attributes and services for establishing how they conform the client's satisfaction. Due to the nature of the problem, the population is dynamic; in fact, it is very usual that the recollection of the information is equivalent to a census. In many occasions, using samples allows completing the information. Therefore the usual statistical inference is not usable. Only descriptive statistical data analysis is of use and the modern tools may be used for deriving coherent analysis of the data. We consider the possibility that the manager will accept the validity of a certain super population model. Then is justified developing studies using methods as principal components for example.

The discussion is illustrated with data collected in Cuban hotels in 2005.

Keywords: Consumer satisfaction index,

\section{Introducción}


La satisfacción de los clientes es el objetivo de los servicio en la actualidad. Para los hoteles este es un problema complejo dada la interrelación de los servicios, las expectativas de los huéspedes y diversos factores exógenos. En los servicios se ha impuesto el uso de índice de satisfacción dl cliente (Consumer Satisfaction Index). Estos toman en cuenta diversos atributos y servicios y les integran en una medida, de la que se extrae la caracterización de la satisfacción de los clientes. En la industria turística es además importante establecer si la satisfacción conlleva la repitencia del turista, al regresar al mismo hotel.

Índices particulares han sido son desarrollados por diversos países e industrias turísticas. Todos se computan al agregar las evaluaciones hechas por los clientes. Los gerentes les utilizan para conocer en que sentido deben mejorar o mantener los servicios que brinda. Sin embargo la agregación de los criterios de los turistas es un modelo que está asociado a grandes sesgos.

Cada turista tiene su propio mecanismo de agregación y este refleja en gran medida lo que considera el nicho de mercado al que pretense. Un turista que está en un nicho que utilizará un hotel de 4 estrellas será más exigente al ir a uno de 3 estrellas que el cliente habitual, por ejemplo. Es necesario evaluar cuan adecuado es la agregación realizada al considerar la evaluación final de la calidad global del servicio y la lealtad del cliente. Los índices no son mas que un a medida que es usada por el gerente para evaluar la calidad del servicio que oferta. Pero si le consideramos desde el punto de vista estadístico este es un parámetros. La problemática de que no se miden variables de naturaleza objetiva sino criterios subjetivos, modelados a partir de fijar escalas de la calidad percibida, complica el enlizarle estadísticamente.

Otro problema complicado es que la población es dinámica. Los huéspedes están por periodos diferentes de tiempo y esto hace que incluso las variables endógenas y exógenas s que influyen en su valoración sean diferentes. De hecho no se trata con muestras sino con censos deficientes. Estos en el mejor de los casos son 
complementados por encuestas especialmente diseñadas. De todos modos tenemos que los índices no son analizables sutilizando métodos estadísticos inferenciales. Consideramos que el gerente puede aceptar un cierto modelo superpoblacional. Bajo este e s posible hacer un estudio utilizando Componentes Principales para determinar que atributos y servicios son importantes al valorar la calidad percibida. Similarmente se puede hacer un estudio de senderos para establecer como se forma la satisfacción y la lealtad de los clientes.

Las secciones 2 y 3 se dedican a hacer una discusión sobre aspectos raigales de los índices de satisfacción del consumidor. Se hace una disección de estos para establecer su relación con un parámetro de una población. En la sección 4 se discute el problema de la población, el censo, la muestra y el cuestionario. En la sección 5 es dedicada a presentar los problemas que aparecen dados la existencia de muchos datos perdidos. Se analiza esto desde el punto de vista de su solución usando métodos de imputación.

Finalmente se ilustra lo discutido al hacer un estudio de las cadenas hoteleras de Cuba con datos recolectados en el 2005. Se hace uso del índice utilizado por el Ministerio d e la Industria Turística. Se establece como el sistema de adición de la satisfacción por servicio no siempre es el adecuado. Para ello se contrasta la satisfacción expresad para cada servicio con la evaluación global y el orden de la satisfacción al calcular el consenso usando un índice de consenso simple. Además se analiza la diferencia entre las expectativas de los huéspedes y su percepción incluyendo los resultados del SERVQUAL GAP.

Las secciones 6 y 7 encaran como las variables utilizadas son evaluables al aceptar un cierto modelo superpoblacional utilizando la consistencia a interna y la varianza promedio extraída. Esto lleva a utilizar Análisis de Componentes Principales para establecer los factores importantes. Este modelo es usado también para realizar un estudio de senderos para diseccionar como se formó la satisfacción y la lealtad en los huéspedes. 


\section{Sobre los Índices de Satisfacción}

Al utilizar un servicio el cliente tiene expectativas y al brindarse estas se tiene una percepción de la calidad .La satisfacción del cliente por el servicio determinará las quejas y la lealtad de este. Esta depende de diversos factores los cuales, una vez identificados, se relacionan en forma causal. La calidad del servicio determina un nivel de satisfacción que determinará la reducción de quejas y el incremento de la lealtad. Considerando la vinculación existente entre la calidad del servicio y la satisfacción del turista, en cuanto a que los niveles de satisfacción del usuario, es evidente la necesidad de medir en forma directa, fácil y económica de en qué medida las expectativas, necesidades y deseos de los clientes son satisfechas. Ciertamente, diversos estudios han identificado las dificultades de objetivizar el concepto de calidad en el servicio. Vea Badri et al. (2005), Jabnoun \& Khalifa (2005), y Landrum et al. (2007), por ejemplo.

Establecer una medición de la calidad en los servicios es bien elusivo y no existe una metodología de universal aceptación. Por ello en diversos contextos su medición está expuesta a multiplicidad de variables exógenas y endógenas en cada caso especifico. Una metodología fue desarrollada en la década de los noventa en los EUA. Allí se desarrolló el llamado. Customer Satisfaction Index Models (ACSI). Este fue elaborado para desarrollar estudios cuantitativos de Mercado. Esta ha servido de marco referencial para desarrollar otros. Básicamente estos utilizan los factores: calidad percibida, expectativas del cliente, valor percibido y satisfacción global del cliente. Índices de satisfacción han sido determinados para acometer estudios en otras latitudes. Algunos de ellos son:

European Customer Satisfaction Index (ECSI). Este es determinado por un modelo que utiliza como constructos: imagen, expectativas del cliente, calidad percibida, 
Satisfacción y lealtad. La causalidad establece que la imagen influye en así las expectativas, estas son afectadas por la calidad percibida. Este modelo varia de acuerdo al servicio evaluado.

El Swiss Index of Customer Satisfaction (SWICS) utiliza los factores: satisfacción del cliente, dialogo con el cliente y lealtad. La calidad está dada por la afectación del dialogo y la lealtad por parte de la satisfacción y que el diálogo afecta la lealtad. En todos los estudios sobre la calidad, en los índices se evidencia que la calidad .percibida es determinada por la satisfacción y esta reduce las quejas e incrementa la lealtad.

\section{El caso hotelero}

En particular el éxito de un hotel reside en la satisfacción de los clientes. El gerente debe comprender que esperan los turistas, la dimensionalidad de la calidad del servicio y su importancia relativa para cada segmento de mercado. Es por ello que deben efectuarse estudios concretos para conocer cuál es la calidad de su serviciaje, desde el punto de vista de la clientela.

La satisfacción es un auto-concepto desde el punto de vista de la psicología. Este está determinado por el sentimiento de placer y bienestar resultantes de la relación entre lo que ambiciona y espera con lo obtenido. Esto es intrínseco a cada ser humano y situación específica.

Los psicólogos consideran como constructos esenciales desde el cliente aquellos que miden:

- Tangibilidad: Existencia de facilidades físicas (equipos, personal, comunicación telefónica y de internet etc.)

- Fiabilidad: Habilidad para brindar el servicio prometido

- Respuesta: Disposición para ayudar el cliente y proveer respuestas rápidas y eficaces. 
- Confiabilidad: Garantía del conocimiento del personal, obtiene un trato cortes, veracidad de su nivel de información.

- Empatía: Vocación para conocer las necesidades y gustos particulares del cliente.

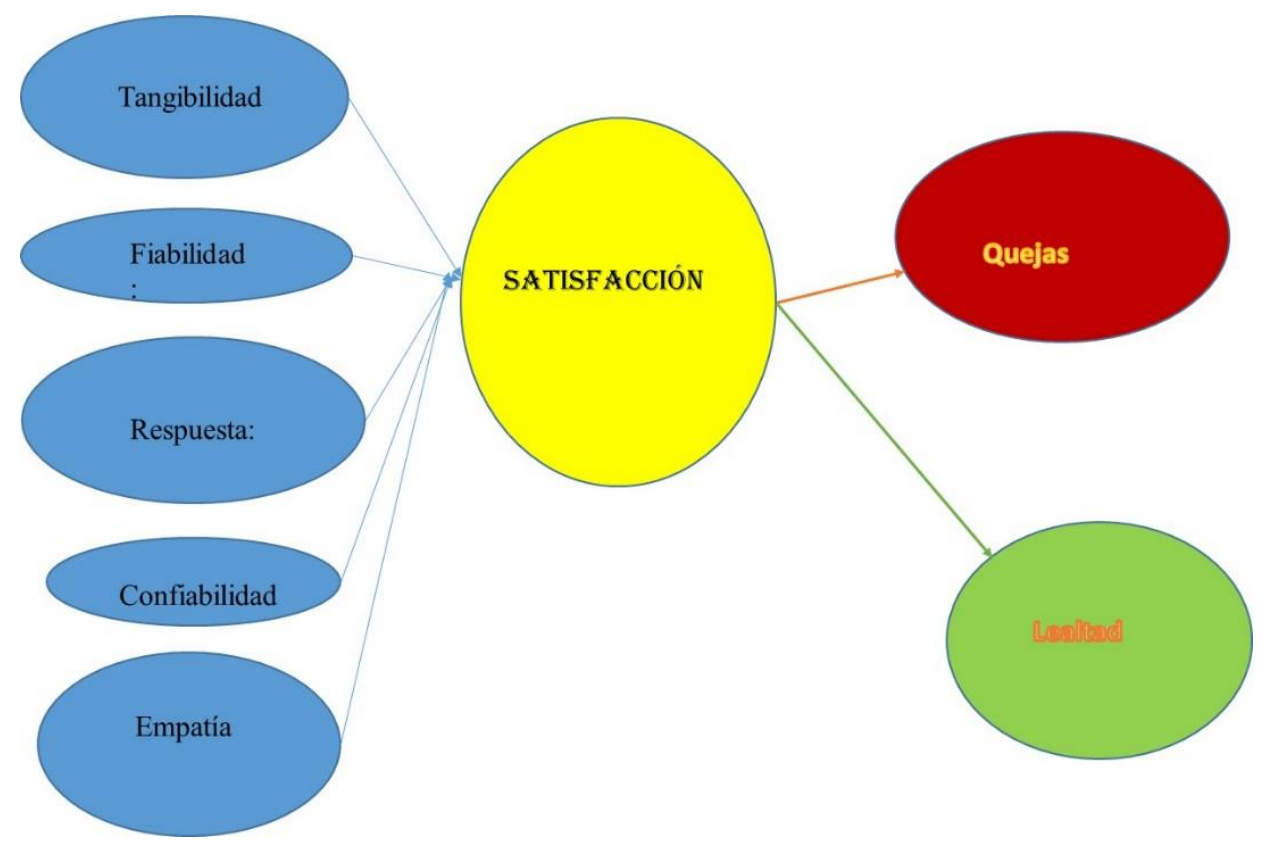

Figura 1. Esquema de la satisfacción desde el punto de vista de la psicología.

Los estudios de satisfacción requieren de obtener información de los clientes sobre factores que midan, de una forma $u$ otra estos constructos.

Parasuraman et al. (1991) afirman que la satisfacción e raigalmente diferente de la calidad del servicio. Esta proviene de cuan satisfecho se está con el servicio obtenido. Indudablemente a mayor calidad mayor satisfacción, pero hay una serie de variables latentes cuyo comportamiento en la satisfacción deben ser identificadas. Identificándoles el gerente sabrá la dirección en la que en mejoramiento de la calidad debe ser enrumbada. 
Así que, el individuo hace una comparación entre lo que aspira y lo que le brindan para establecer su nivel de satisfacción.

Por ello no se puede considerar la calidad del servicio como una medida absoluta. Ella es relativa, pues no depende solo de la eficacia de lo que se ofrece sino del par dialéctico expectativa-percepción de cada cliente.

Puede considerarse que la satisfacción es una medida de corto plazo pues se expresa por las transacciones expectativas-percepciones. La calidad de los servicios es el resultado de un movimiento a largo plazo, producto de un desempeño del servicio brindado.

La cadena hotelera Ritz-Carlton ha definido su patrón de calidad por la eficiencia en atraer, satisfacer, exceder y retener a todos los clientes internos y externos, haciendo las cosas a la medida de las características del cliente.

Una herramienta de común aceptación en estudios de calidad hotelera es SERVQUAL. Esta permite comprender como los diversos factores afectan la calidad en el servicio de hoteles. Ella es de naturaleza multidimensional. SERVQUAL es un modelo que sirve como base para medir la calidad de los servicios hoteleros. Los investigadores de la calidad hotelera al estudiar la calidad de los servicios le modifican y adaptan.

Un estudio que ha fijado pautas en su uso es el de Akbaba (2006). En él se analizaron las expectativas y percepciones de la calidad del servicio usando 29 indicadores, determinando que la tangibilidad de los servicios era el factor más indicativo de la valoración del cliente.

La demanda de mejorar el servicio se ha visto incrementada dado el desarrollo de la complejidad del sector. Vea Wu et al. (2012). Esto ha sido el motor impulsor del gran número de investigaciones sobre la claridad de los servicios hoteleros. La 
globalización ha llevado a que estos estudios sean necesarios en todo el mundo de los servicios.

En la industria hotelera la calidad es expresada en términos de las expectativas del huésped-cliente y la percepción que calidad tenga. Por ello la competividad de los hoteles se basa en la mejora incesante de la calidad de sus servicios. La percepción de una calidad mejorada estimula la lealtad de los clientes habituales y la preferencia de los nuevos.

Cada investigador debe, al utilizar SERVQUAL, examinar las dimensiones a incluir en su estudio. Si el hotel se inserta en el mercado internacional deberá tomar en cuenta la inclusión de dimensiones significativas que están en incluidas en los estudios de los países de los emisores de turismo.

\section{Muestra cuestionario}

En todo estudio concreto de satisfacción-calidad es necesario conocer los criterios de los clientes. El instrumento más usual y recomendable en los hoteles es utilizar un cuestionario que es llenado por el huésped en forma voluntaria. Con los datos se deben llevar a cabo varios análisis del arsenal estadístico. Uno de ellos es estudiar la relación explicativa de la relación huésped-percepción del servicio del hotel. Una comparación entre estas variables debe ser realizada. Si estas coincidieren la gerencia se dará por satisfecha con su labor. Si la diferencia entre expectativas y satisfacción es negativa debe estudiar sus causas para mejorar la calidad en la dirección indicada. Si fuera positiva su política es triunfadora y por tanto lograra la lealtad de los huéspedes.

Esta descripción brindará una panorámica que hace parecer sencillo el evaluar un estudio del servicio.

Lo más común es dejar un cuestionario en los cuartos. Este puede variar a partir de los criterios de la gerencia. Los más sencillos solo esperan una valoración en 
una k-escala de Likert de la satisfacción del huésped con ciertos servicios. En otros casos puede ser algo más sofisticado y ocasionalmente harán alguna entrevista personal. Del análisis de los cuestionarios se espera diagnosticar las fallas del sistema para mejorar la calidad.

Si miramos el problema desde un punto estadístico se presentan varios problemas complejos que hacen dudable la efectividad de los análisis basados eses datos.

\section{La población.}

Los modelos de la estadística basada en el principio del muestreo repetido parten de que la población es fija. Sin embargo en los estudios hoteleros esta varía dinámicamente. Por tanto no podemos suponer que tenemos una población finita sometida a los mismos efectos exteriores y que esta es $U=\{1, \ldots, N\}$. Quizás lo mas apropiado será considera un población de habitaciones las cuales emiten un dato varias veces en un periodo. Siguiendo este modelo habría que promediar lo que ocurre en cada uno de ellos antes de pasar a hacer un análisis a nivel del hotel y sus servicios. Si consideramos la existencia de información suficiente para ajustar algún modelo Bayesiano se estaría infiriendo sobre el mecanismo que genera las poblaciones. Lamentablemente la naturaleza del problema hotelero hace difícil que los ejecutivos sean capaces de ponerse de acuerdo sobre que distribución apriorística describe los resultados. Utilizar un método de simulación del tipo Gibss-sampler puede ser adecuado, pero el problema inferencial asociado a la calidad lo que busca es una caracterización de cómo afecta la calidad en la satisfacción. Esto haría más difícil obtener los factores latentes pues se carece de una modelación teórica de tales problemas.

En todo caso el interés de la gerencia no està en que pasò con los huéspedes sino cual es el criterio de estos sobre la calidad para ejorar en 
el sentido de las expectativas de los huéspedes de los nichos de mercado hacia oos cuales se enrumba su servicio.

\section{La muestra}

La muestra misma es afectada por el carácter "voluntario" de los respondientes. La calidad de la información es afectada por la existencia usual de un gran número de no respuestas. En la práctica no se cuenta con una muestra sino de un ineficiente censo de la población, cualquiera sea su definición.

\section{Los modelos}

El análisis exploratorio de datos se basa en modelos dudables dado lo discutido anteriormente.

\section{Los datos.}

Cada respondiente debe llenar un cuestionario que posee $\mathrm{m}$ ítems con $\mathrm{k}$ posibles respuestas. Por tanto se cuenta con mk! posibles resultados del reporte. En realidad la respuesta a cada ítem es un rango. Por tanto las hipótesis sobre continuidad no son válidas. Dada la naturaleza de las respuestas empieza a ser dudable el uso de medias.

En los primeros pasos en los estudios de estadística nos dicen que es mejor la mediana cuando trabajamos con rangos. Sin embargo nadie hace uso de ella en los estudios hoteleros en la literatura. Pero además quizás lo más importante para la gerencia es la moda. El gerente esta más interesado en cual es el criterio más frecuente sobre un factor del ítem. Complementará este al compararle con la mediana y la media, si es suficientemente hábil. Calificar pues el servicio usando medias conlleva a un mal uso de las estadísticas elementales. Dado que no hay un modelo 
probabilístico no es validos hacer inferencias y lo que se tiene es que coniar en la habilidad de discernir lo que dicen los datos. El estadístico aconseja y el gerente infiere.

\section{Los datos perdidos}

Dada la naturaleza del mecanismo de la pérdida de los datos por no respuestas es necesario reparar en este hecho y de una u otra forma, o tomarle en cuenta.

Los Datos perdidos por no respuestas pueden venir dados por:

No reporte por parte de los huéspedes.

Entonces hay dos estratos de tamaños $N_{1}$ y $N_{2}$

i) Estrato 1 formado por los huéspedes que responden.

ii) Estrato 2 formado por los huéspedes no responden

En muchos casos la no respuesta responde a problemas de tipo administrativo. Vale estimar las respuestas de alguna forma!!!

- Hay No-respuestas de huéspedes debido a negativas a responder o a no contactarlas.

- Hay Ítem no-respuesta pues en algunos huéspedes o dan toda la información.

- No concordancia entre los records para los respondientes rechazos, omisiones de los entrevistadores, Pérdida Accidental de los datos o del cuestionario. (llovió se mojó la planilla y se hizo ilegible) etc.

- Es de señalar que la NR es un fenómeno multivariado pues es el resultado de una serie de fenómenos concurrentes en el desarrollo de la encuesta. 
Puede decidirse Submuestrear los No-respondientes. En tal caso puede enviarse por correo a algunos el cuestionario o utilizar el correo electrónico. Para ello se selecciona una submuestra de los no respondientes.

Puede decidirse usar Los datos obtenidos. En tales casos se descarta la información no obtenida. Esto es valido si la media de respondientes y la de los no respondientes son tales que

\section{$\bar{X}_{\text {responden }} \triangleq \bar{X}_{\text {no-responden }}$}

Por tanto solo si las respuestas de los no respondientes son "iguales" a la de los que responden no considerar las no respuestas es un riesgo de cometer un gran error en la estimación!!

Puede decidirse Imputar los datos faltantes. Al decidir esto los no-respondientes son reemplazados asignándoles un cierto valor a su respuesta. Vamos en tal caso a utilizar la información recabada... y/o hacer substituciones inteligentes de los datos faltantes. Una variante es considerar que hay un mecanismo determinístico que modela la existencia de no respuestas. Entonces los que no responden están determinados de antemano al hacer la encuesta aunque el muestrista no sabe quienes son.

Si se trabaja con proporciones y $n_{1}$ es grande

\section{$p_{1}=$ numero de respuestas positivas $/ n_{1}$}

Al haber NR tenemos que una cota inferior del intervalo de confianza, si decidimos usarles, para la proporción poblacional $\mathrm{P}$ es 


$$
\begin{aligned}
& p_{\text {inf }}=W_{1}\left(p_{1}-z_{1-\frac{\alpha}{2}} \sqrt{\frac{p_{1}\left(1-p_{1}\right)}{n_{1}}}\right)+W_{2} \times 0=W_{1}\left(p_{1}-z_{1-\frac{\alpha}{2}} \sqrt{\frac{p_{1}\left(1-p_{1}\right)}{n_{1}}}\right) \\
& p_{\text {sup }}=W_{1}\left(p_{1}-z_{1-\frac{\alpha}{2}} \sqrt{\frac{p_{1}\left(1-p_{1}\right)}{n_{1}}}\right)+W_{2} \times 1=W_{1}\left(p_{1}-z_{1-\frac{\alpha}{2}} \sqrt{\frac{p_{1}\left(1-p_{1}\right)}{n_{1}}}\right)+W_{2}
\end{aligned}
$$

Como $\mathrm{P}_{2}$ no puede ser cero y 1 al mismo tiempo podemos hacer más pequeño el IC

Si se acepta la existencia de un modelo Probabilístico que modela las no respuestas cada individuo en la población está asociado a un mecanismo que genera que este entre los respondientes o no. Este genera una variable Binomial

$B(i)=$ número de veces en que la unidad i respondería bajo las condiciones de la encuesta en desarrollo

Hay Patrones de no respuesta

- Univariadas

- Multivariadas

- Monótonas

- Generales

- "Matching" de los ficheros

- Factores Latentes

- Parámetros y el paradigma de Bayes

Un procedimiento de imputación es el llamado de Imputación simple. Entre ellos se encuentran 
- Hot deck

- Cold deck

- Substitución por la media

- Substitución basada en una Regresión

- Substitución basada en una regresión estocástica.

- Imputación Aleatoria.

Los métodos de "Hot deck" son sobre todo muy usados en el caso de la variables categóricas, que es el que nos compete.

Por otra parte los Mecanismos que generan pérdida de datos son:

- Pérdida completamente aleatoria (Missing Completely At Random), la denotamos MCAR. (La probabilidad de pérdida no depende nada).

- Perdida aleatoria (Missing At Random) la denotamos MAR. (La probabilidad de pérdida no depende del valor perdido de la variable de interés pero si puede depender de otras variables de respuesta)

- Pérdida aleatoria, (Not Missing at Random) la denotamos NMAR. (La probabilidad de pérdida depende del valor de la variable no medida) -

\section{Índices de satisfacción y un estudio hotelero}

A la larga los índices no son sino algún tipo de promedio de los atributos. En su creación se debe hacer estudios estadísticos a sabiendas de que no se pueden hacer inferencias. Por ello usamos algún método del análisis de regresión múltiple, para detectar las posibles colinealidades, del análisis factorial u otra técnica similar para tener idea de la variabilidad de los factores. Lo ideal para el gerente es usar solo un índice pero en general es recomendable determinar varios en función de los servicios agregados. De todos modos todo inicie no es sino un valor numérico único. Esto puede llevar a malinterpretaciones por trabajar 
con una media. Valdría la pena estudiar este problema con más detalle. Para ello utilizaremos un estudio desarrollado en Cuba usando un índice de adición simple.

Utilizamos viejos datos de 181 hoteles cubanos. El método usado por el Ministerio de Turismo, en estado desarrollado en el 2005, fue el hacer una agregación de las calificaciones dadas POR los huéspedes. Su objetivo fue hacer un diagnostico y no el de mejorar la calidad. Por ello agruparon hoteles por cadena s y provincias. En esta presentación expondremos el análisis realizado en provincias con más de 3 cadenas hoteleras. El primer error fue el de agrupar los hoteles por cadenas $\sin$ hacer distinción de su nivel. El otro fue el de no hacer ponderación de los servicios.

Los servicio evaluados fueron los servicios de recepción, la calidad de las habitaciones, la satisfacción con la animaciòn-recreaciòn, el trato y ofertas en al tiendas, la limpieza, el manejo del idioma la calidad del servicio. Ademes se dio una evaluación global al hotel. La información recabada por cadena hotelera llevó a los siguientes resultados

Tabla 1. Evaluación de los servicios en Pinar del Rio

\begin{tabular}{|l|l|l|l|l|}
\hline Servicio & & & cadena & \\
\hline & $\begin{array}{l}\text { Gran } \\
\text { Caribe }\end{array}$ & Cubanacán & Isla Azul & Gaviota \\
\hline Recepción & $4(3)$ & $4,44(2)$ & $4,67(1)$ & $1(4)$ \\
\hline Habitaciones & $4,9(1)$ & $3,92(3)$ & $4(2)$ & $2(4)$ \\
\hline $\begin{array}{l}\text { Animaciòn- } \\
\text { Recreaciòn }\end{array}$ & $4,6(1)$ & $3,81(3)$ & $4(2)$ & $1(4)$ \\
\hline Tiendas & $0(4)$ & $3,94(1)$ & $2(2)$ & $1(3)$ \\
\hline Limpieza & $4(2)$ & $3,91(3)$ & $4,33(1)$ & $1(4)$ \\
\hline Dominio de & $0(4)$ & $4,08(2)$ & $4,33(1)$ & $2(3)$ \\
\hline
\end{tabular}




\begin{tabular}{|c|c|c|c|c|}
\hline idiomas & & & & \\
\hline $\begin{array}{l}\text { Calidad del } \\
\text { servicio }\end{array}$ & $5(1)$ & $4,12(3)$ & $4,33(2)$ & 1(4) \\
\hline $\begin{array}{l}\text { Evaluación } \\
\text { global }\end{array}$ & $4(3)$ & $4,04(2)$ & $4,27(1)$ & $1(4)$ \\
\hline $\begin{array}{l}\text { Índice de } \\
\text { consenso } \\
\text { simple }\end{array}$ & 2 & 2 & 1 & 4 \\
\hline
\end{tabular}

Como se ve la adición es poco confiable si se considera importante el dominio de idiomas y la oferta en tiendas.

Tabla 2. Evaluación de los servicios en La Habana

\begin{tabular}{|l|l|l|l|l|l|l|}
\hline Servicio & & & cadena & & & \\
\hline & $\begin{array}{l}\text { Gran } \\
\text { Caribe }\end{array}$ & Cubanacán & $\begin{array}{l}\text { Isla } \\
\text { Azul }\end{array}$ & Gaviota & Habaguanex & Otros \\
\hline Recepción & $4,16(4)$ & $4,25(2)$ & $3,39(6)$ & $4,0(5)$ & $4,2(3)$ & $5(1)$ \\
\hline Habitaciones & $3,94(3)$ & $4,02(1)$ & $3,48(5)$ & $3,95(2)$ & $3,87(4)$ & $0(6)$ \\
\hline $\begin{array}{l}\text { Animaciòn- } \\
\text { Recreaciòn }\end{array}$ & $3,37(4)$ & $3,93(2)$ & $3,27(5)$ & $4(1)$ & $3,71(3)$ & $0(6)$ \\
\hline Tiendas & $3,62(4)$ & $3,77(2)$ & $2,5(5)$ & $3,79(1)$ & $3,67(3)$ & $0(6)$ \\
\hline
\end{tabular}




\begin{tabular}{|l|l|l|l|l|l|l|}
\hline Limpieza & $3,97(5)$ & $4,05(4)$ & $3,61(6)$ & $4,11(3)$ & $4,39(2)$ & $5(1)$ \\
\hline $\begin{array}{l}\text { Dominio de } \\
\text { idiomas }\end{array}$ & $4,23(3)$ & $4,26(2)$ & $2,71(5)$ & $4,23(3)$ & $4,55(1)$ & $0(6)$ \\
\hline $\begin{array}{l}\text { Calidad del } \\
\text { servicio }\end{array}$ & $4,06(4)$ & $4,19(3)$ & $3,33(6)$ & $4(5)$ & $4,36(2)$ & $5(1)$ \\
\hline $\begin{array}{l}\text { Evaluación } \\
\text { global }\end{array}$ & $4,01(4)$ & $4,10(2)$ & $3,27(6)$ & $4,02(3)$ & $4,1(4)$ & $5(1)$ \\
\hline $\begin{array}{l}\text { Índice de } \\
\text { consenso } \\
\text { simple }\end{array}$ & 4 & 1 & 6 & 3 & 2 & 5 \\
\hline
\end{tabular}

Como se ve la adición no es lineal simple pues "otros" obtiene tres calificaciones máximas y 4 mínimas y sin embargo obtiene la máxima calificación global.

Tabla 3. Evaluación de los servicios en Varadero

\begin{tabular}{|l|l|l|l|l|l|}
\hline Servicio & & & cadena & & \\
\hline & $\begin{array}{l}\text { Gran } \\
\text { Caribe }\end{array}$ & Cubanacán & Isla Azul & Gaviota & Otros \\
\hline Recepción & $3,8(4)$ & $4,2(1)$ & $3,88(3)$ & $4,14(2)$ & $3(5)$ \\
\hline Habitaciones & $3,35(3)$ & $3,99(1)$ & $3,06(4)$ & $3,95(2)$ & $2(5)$ \\
\hline Animaciòn- & $3,38(3)$ & $4,03(1)$ & $2,73(4)$ & $3,88(2)$ & $1(5)$ \\
\hline
\end{tabular}




\begin{tabular}{|l|l|l|l|l|l|}
\hline Recreaciòn & & & & & \\
\hline Tiendas & $3,29(3)$ & $3,56(1)$ & $2,88(4)$ & $3,43(2)$ & $1(5)$ \\
\hline Limpieza & $3,57(3)$ & $4,03(1)$ & $3,15(4)$ & $3,98(2)$ & $1,5(5)$ \\
\hline $\begin{array}{l}\text { Dominio de } \\
\text { idiomas }\end{array}$ & $3,91(3)$ & $4,27(1)$ & $3,42(4)$ & $4,02(2)$ & $2(5)$ \\
\hline $\begin{array}{l}\text { Calidad del } \\
\text { servicio }\end{array}$ & $3,72(3)$ & $4,24(1)$ & $3,38(4)$ & $4,15(2)$ & $3(5)$ \\
\hline $\begin{array}{l}\text { Evaluación } \\
\text { global }\end{array}$ & $3,56(3)$ & $4,08(1)$ & $3,23(4)$ & $3,95(2)$ & $2,46(5)$ \\
\hline $\begin{array}{l}\text { Índice de } \\
\text { consenso } \\
\text { simple }\end{array}$ & 3 & 1 & 4 & 2 & 5 \\
\hline
\end{tabular}

En Varadero el comportamiento de las cadenas es uniforme. Cubanacán es la mejor y "otros" la peor. Hay una clara agregación lineal.

Tabla 4. Evaluación de los servicios en Jardines del Rey

\begin{tabular}{|l|l|l|l|l|}
\hline Servicio & & cad & ena & \\
\hline & $\begin{array}{l}\text { Gran } \\
\text { Caribe }\end{array}$ & Cubanacán & Isla Azul & Gaviota \\
\hline Recepción & $4,41(3)$ & $4,43(2)$ & $5(1)$ & $4,35(4)$ \\
\hline
\end{tabular}

REDMARKA UIMA-Universidad de A Coruña - CIECID 


\begin{tabular}{|l|l|l|l|l|}
\hline Habitaciones & $4,04(3)$ & $3,51(4)$ & $5(1)$ & $4,13(2)$ \\
\hline $\begin{array}{l}\text { Animaciòn- } \\
\text { Recreaciòn }\end{array}$ & $4,08(1)$ & $4(2)$ & $4(2)$ & $3,7(4)$ \\
\hline Tiendas & $4,1(2)$ & $4,09(3)$ & $2(4)$ & $4,17(1)$ \\
\hline Limpieza & $3,47(1)$ & $3,39(2)$ & $1(4)$ & $3,28(3)$ \\
\hline $\begin{array}{l}\text { Dominio de } \\
\text { idiomas }\end{array}$ & $4,19(2)$ & $3,87(4)$ & $5(1)$ & $4,17(3)$ \\
\hline $\begin{array}{l}\text { Calidad del } \\
\text { servicio }\end{array}$ & $4,52(2)$ & $4,39(3)$ & $5(1)$ & $4,11(4)$ \\
\hline $\begin{array}{l}\text { Evaluación } \\
\text { global }\end{array}$ & $4,56(1)$ & $4,15(3)$ & $3,86(4)$ & $4,28(2)$ \\
\hline $\begin{array}{l}\text { Índice de } \\
\text { consenso } \\
\text { simple }\end{array}$ & 1 & 3 & 2 & 3 \\
\hline
\end{tabular}

Para Jardines del Rey la adición parece ser lineal. Todo eso hace dudable la efectividad del índice utilizado. A pesar de las deficiencias de la toma de información y del índice podemos objetivizar los resultados. Al partir de las valoraciones de las cadenas (expectativas) y la percepción de los huéspedes se obtuvo:

Tabla 5. Evaluación de los servicios en La Habana

\begin{tabular}{|l|l|l|l|}
\hline Servicio & & & \\
\hline & Expectativa & Percepción & Percepciòn- Expectativa \\
\hline
\end{tabular}




\begin{tabular}{|l|l|l|l|}
\hline Recepción & 5 & 4,12 & $-0,88$ \\
\hline Habitaciones & 5 & 3,9 & $-1,1$ \\
\hline Animaciòn-Recreaciòn & 5 & 3,7 & $-1,3$ \\
\hline Tiendas & 4 & 3,85 & $-0,15$ \\
\hline Limpieza & 4 & 3,46 & $-0,54$ \\
\hline Dominio de idiomas & 4 & 4,04 & 0,04 \\
\hline Calidad del servicio & 4 & 4,11 & 0,11 \\
\hline SERVQUAL GAP & 4 & 3,98 & $-0,2$ \\
\hline
\end{tabular}

Como se ve el servicio es deficiente en casi todos los servicios pero sin embargo el Gap es pequeño.

Nosotros analizando el problema proponemos que la confección y análisis de los datos este regido por el esquema en la figura siguiente 


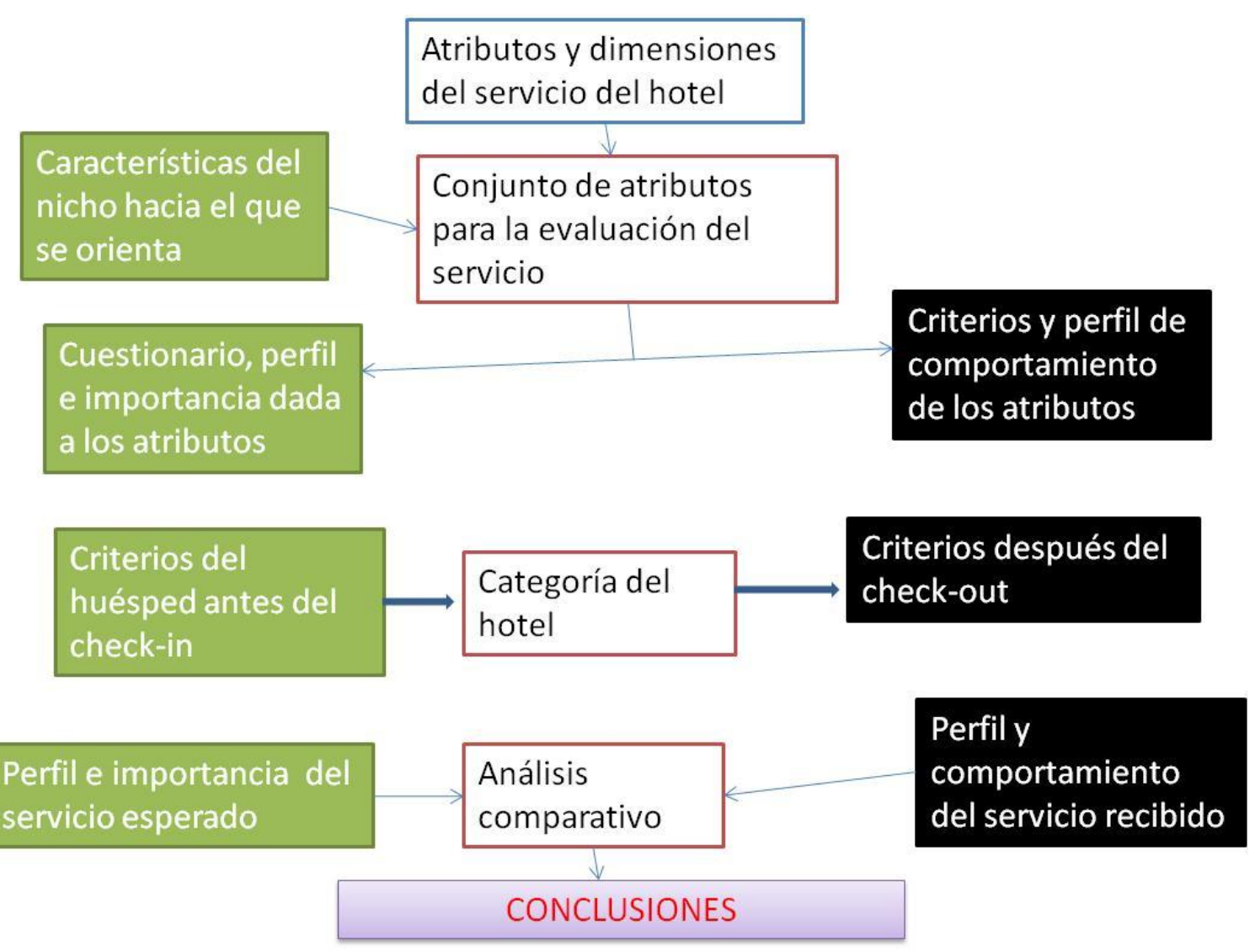

Figura 2. Atributos y dimensiones en un hotel

Entonces el trabajo de captación de información y análisis respondería con un trabajo como el esquematizado en la figura siguiente 

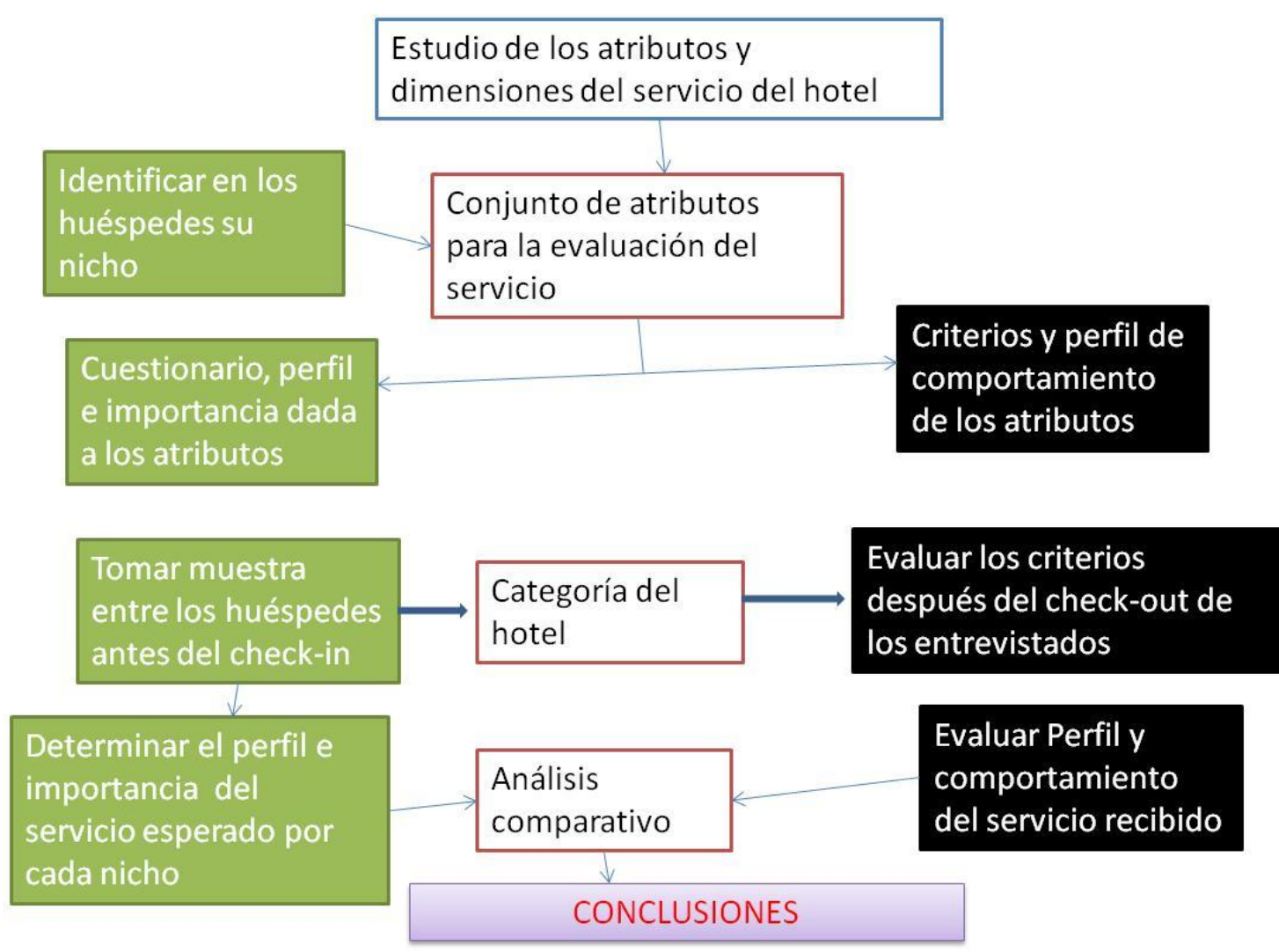

Figura 3. Esquema de un estudio de atributos y dimensiones en un hotel

\section{Fiabilidad de las variables}

Todos van a depender de la fiabilidad de las variables consideradas. En nuestro estudio se usó un modelo basado en ecuaciones estructurales, calculando la consistencia interna de los coeficientes. Sea $\lambda_{i}$ el factor de pesos (loading) del ítem i, la consistencia interna está dada por

$$
\text { Consistencia Interna }=C I=\frac{\left(\sum_{i} \lambda_{i}\right)^{2}}{\left(\sum_{i} \lambda_{i}\right)^{2}+\sum_{i} V\left(\varepsilon_{i}\right)}, \quad V\left(\varepsilon_{i}\right)=1-\lambda_{i}^{2}
$$


$\mathrm{Si} \mathrm{Cl}>0,7$ es satisfactoria el nivel de consistencia interna.

La validez factorial y la discriminante se basan en los $\lambda_{i}$.

El análisis confirmatorio se lleva a cabo analizando la varianza promedio extraída

Varianza promedio extraida $=A V E=\frac{\sum_{i} \lambda_{i}^{2}}{\sum_{i} \lambda_{i}^{2}+\sum_{i} V\left(\varepsilon_{i}\right)}, \quad V\left(\varepsilon_{i}\right)=1-\lambda_{i}^{2}$

En la diagonal de la próxima tabla esta $C=\sqrt{A V E}$. Si $\mathrm{C}<$ coeficientes de correlación tenemos la validez del discriminante.

\begin{tabular}{|c|c|c|c|c|c|c|c|}
\hline Servicio & & & cadena & & & & \\
\hline & $\begin{array}{l}\text { Recepci } \\
\text { ón }\end{array}$ & $\begin{array}{l}\text { habitacion } \\
\text { es }\end{array}$ & $\begin{array}{l}\text { Animaci } \\
\text { ón } \\
\text { recreaci } \\
\text { ón }\end{array}$ & $\begin{array}{l}\text { tienda } \\
\mathrm{s}\end{array}$ & $\begin{array}{l}\text { limpiez } \\
\text { a }\end{array}$ & $\begin{array}{l}\text { Domini } \\
\text { o de } \\
\text { idioma } \\
\text { s }\end{array}$ & $\begin{array}{l}\text { Calida } \\
\text { d del } \\
\text { servici } \\
\text { o }\end{array}$ \\
\hline Recepción & 0,96 & & & & & & \\
\hline $\begin{array}{l}\text { Habitacion } \\
\text { es }\end{array}$ & 0,79 & 0,93 & & & & & \\
\hline $\begin{array}{l}\text { Animaciòn- } \\
\text { Recreaciòn }\end{array}$ & 0,67 & 0,13 & 0,31 & & & & \\
\hline Tiendas & 0,12 & 0,32 & 0,11 & 0,65 & & & \\
\hline Limpieza & 0,23 & 0,75 & 0,34 & 0,91 & 0,85 & & \\
\hline $\begin{array}{l}\text { Dominio } \\
\text { de idiomas }\end{array}$ & 0,37 & 0,21 & 0,58 & 0,88 & 0,60 & 0,52 & \\
\hline Calidad del & 0,77 & 0,19 & 0,32 & 0,53 & 0,43 & 0,84 & 0,94 \\
\hline
\end{tabular}




\begin{tabular}{|l|l|l|l|l|l|l|l|}
\hline servicio & & & & & & & \\
\hline $\begin{array}{l}\text { Evaluación } \\
\text { global }\end{array}$ & 0,85 & 0,87 & 0,42 & 0,28 & 0,94 & 0,79 & 0,87 \\
\hline
\end{tabular}

Note que solo son superados todos los coeficientes de correlación para recepción y habitaciones y limpieza. Por tanto son ellos los que poseen poder discriminante y los mas importantes para la evaluación global.

\section{¿Hacer un estudio usando un modelo estadístico?}

Ante la no posibilidad de utilizar la inferencia estadística dada la no aleatoriedad de los datos observados una solución es considerar valida la existencia de una ecuación estructural

$\eta=B \eta+\Gamma \xi+\zeta$

En esta hay $m$ variables latentes endógenas que determinan el vector $\eta$. Estas son función de las dadas y también de la $q$ variables latentes exógenas en $\xi$. La porción no explicada de las variables endógenas (los errores en la ecuación), tiene una matriz de varianzas y covarianzas

$\Psi=E\left[\zeta \zeta \zeta^{\prime}\right]$.

En general $\eta$ y $\xi$ son consideradas variables endógenas y exógenas respecto al tipo de variables empleadas para estimar la ecuación estructural. Respectivamente porque los errores de medición son constructos hipotéticos imposibles de medir. Variables observables sin errores de medición 0 inconsistencias pueden ser incluidas sin perder en generalidad. Dos modelos representan la relaciones entre las variables latentes $\eta \mathrm{y} \xi$, y sus indicatrices $x, y$ 
$x=\Lambda_{x} \xi+\delta$

$y=\Lambda_{y} \eta+\varepsilon$

Tenemos que $x$ es un conjunto de $p$ variables observadas manifiestas variables consideradas como indicatrices de las variables explicativas $\xi$ y $\delta$ es un vector que representa el error de medición en $x$.

$\Lambda_{x}$ es una matriz de $(p \times m)$ y consiste de los pesos dados por la regresión de $x$ sobre $\xi$. Esta es referida como las ecuaciones de medición. Similarmente se pueda definir la estructura de las variables endógenas latentes de la ecuación equivalente de medición. Los errores de medición $\delta$ y $\varepsilon$ están incorrelacionados con $\eta, \xi$ y $\zeta$ pero que pueden estarlo entre si.

Al aceptar este enfoque el decisor fija, usando hipótesis sobre el problema los parámetros desconocidos de $B, \Gamma, \Psi, \Lambda_{x}, \Lambda_{y}$. Es el llamado análisis factorial confirmatorio o el modelo restringido. Para escalar las variables latentes (métrica) e igualarle a la de las variables observadas. De ahí el sentido del término "variable latente" es requerido escalar algunos de los factores de carga o pesaje (loading factor).

Usando el modelo LISREL y se derivó la siguiente figura 


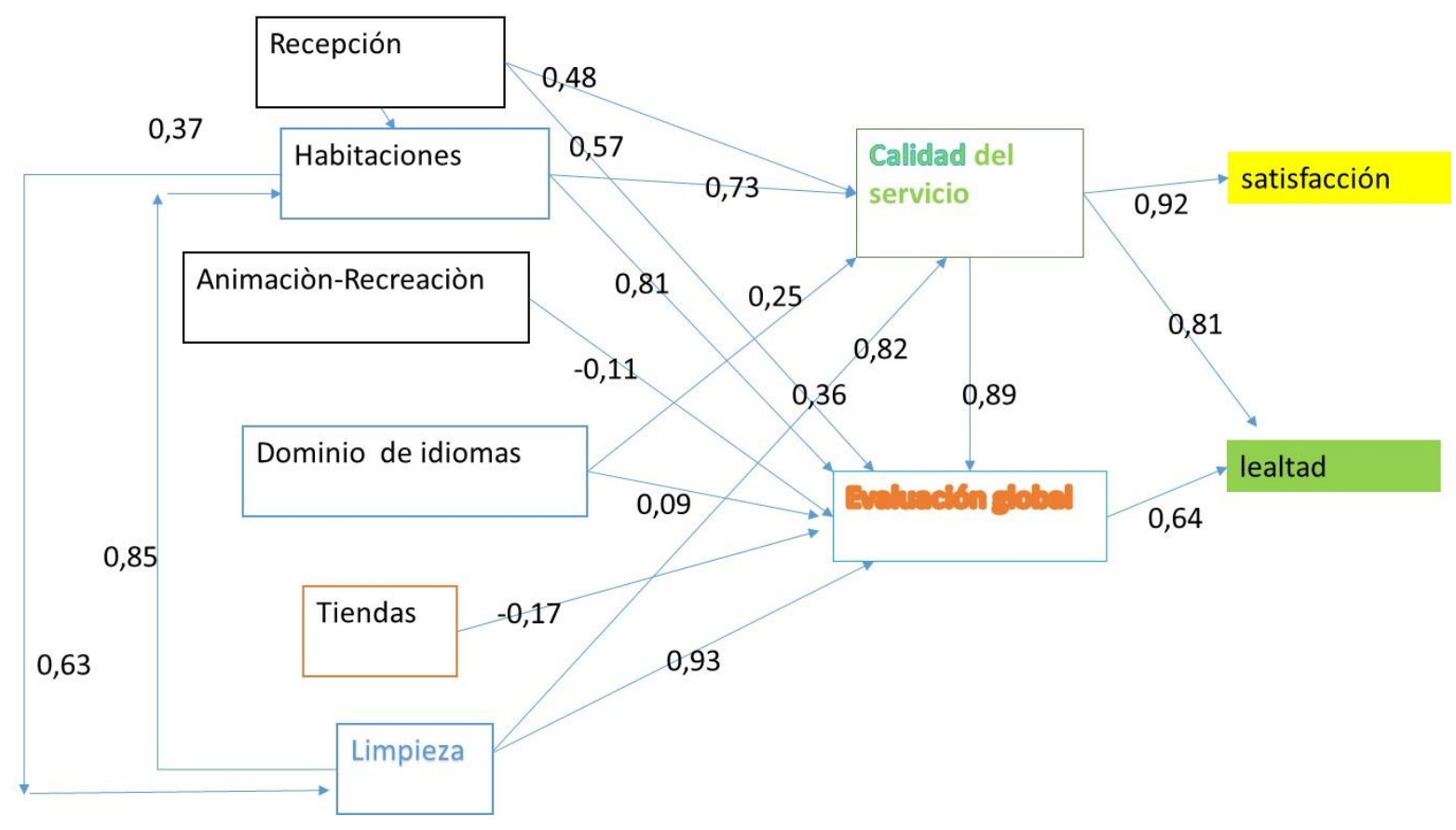

Figura 4. El resultado del estudio de senderos

Como se ve el camino a la calidad del servicio y la evaluación global más fuerte es el que va de la atención recibida en la reacción a la calidad de habitaciones a la limpieza. Moverse de ahí a la lealtad es lo mejor. Los caminos a tres de la evaluación global es un sólido camino pero es más fuerte. La conexión directa más fuerte con la calidad del servicio y la evaluación global es la limpieza. La calidad del servicio determina un sólido camino a la evaluación global, pero al usar el camino a la lealtad a través de esta es menos fuerte que buscarla directamente. Sorpresivamente la evaluación global no se conecta con satisfacción. Solo lo hace a través de la calidad del servicio.

Referencias 
Andrew B. \& SELAMAT A, (2012). Systematic Literature Review of Missing Data Imputation Techniques for Effort Prediction. IPCSIT vol.45 (2012) IACSIT Press, Singapore

Arnould, E. J.; Price, L. (1993): River Magic: Extraordinary Experience and the Extended

Service Encounter, Journal of Consumer Research, 20 24-45.

Ary, D.; Jacobs, L.C.; Razavieh, (2010): A. Introduction to Research in Education. Wadsworth/Thomson Learning, Belmont.

Asubonteng, P.; McCleary, K.J.; Swan, J.E. (1996): SERVQUAL revisited: a critical review of service quality, Journal of Services Marketing, 10, 62-81.

Bernini, C. Y S. Bitner, M.J.; Booms, B.H.; Tetreault, M.S. (1990): The service encounter: diagnosing favourable and unfavourable incidents, Journal of Marketing, 54, 71-84.

Blešiš, I.; Ivkov-Džigurski, A.; Dragin, A.; Ivanoviš, L.; Panteliš, M. (2011): Application of Gap model in the researches of hotel services quality, Turizam, 15, 40-52.

Boulding, W.; Kalra, A.; Staelin, R.; Zeithaml, V.A. (1993): A dynamic process model ofservice quality: from expectations to behavioral intentions, Journal of Marketing

Research, 30,. 7-27.

Buttle, F. (1996): SERVQUAL: review, critique, research agenda, European Journal ofMarketing, 30, 8-32. 
Cagnone (2007): Multigroup-Multiwaves Lisrel Modeling. In Tourist Satisfaction Analysis. StatisticA, LXVII, 235-254

Campos, D. F., Marodin, T. G. (2003): Perceptions of Quality and Expectations of Hotel Service. Journal of Operations and Supply Chain Management 5, 82 - 99.

Crompton, J.L.; Love, L.L.(1995): The Predictive Value of Alternative Approaches to

Evaluating Quality of a Festival, Journal of Travel Research, Summer, 11-24.

Dixit S.K. (2013): A Study Of Guest's Expectation And Perception Of Hotel Service Quality: Case Of Khajuraho, India. Enlightening Tourism. A Pathmaking Journal, $3, .125-141$.

Edwin, A. M. (2009): Ponderación de la muestra y tratamiento de valores faltantes en las variables de ingreso en la Encuesta Permanente de Hogares. República Argentina, Ministerio de Economía y Finanzas Públicas, Instituto Nacional de Estadística y Censos. B. Aires.

Ekinci, Y.; Riley, M.; Fife-Schaw, C. (1998): Which school of thought? The dimensions of resort hotel quality, International Journal of Contemporary Hospitality Management, 10, 63-7.

Fick, G.R.; Ritchie, J.R.B. (1991): Measuring service quality in the travel and tourism industry, Journal of Travel Research, 30, 2-9,.

Gandolfo Dominici , Rosa Guzzo (2012): Customer Satisfaction in the Hotel Industry: A Case Study from Sicily Measuring Service Quality Dimensions: An Empirical Analysis of Thai Hotel Industry. International Journal of Business Administration 3, 52-63 
Getty, G.M.; Thompson, K.N. (1994): The Relationship between Quality, Satisfaction and

Recommending Behaviour in Lodging Decisions, Journal of Hospitality and Leisure Marketing, 2,33-21.

Geva, A.; Goldman, A. (1991): Satisfaction Measurement in Guided Tours, Annals ofTourism Research, 18, 177-185.

Kandampully, J.; Hu, H.H. (2007): Do hoteliers need to manage image to retain loyal customers? International Journal of Contemporary Hospitality Management, 19, 435-443.

Little, R.J.A. Y Rubin, D.B., (2002), Statistical Analysis With Missing Data, Wiley \& Sons.

Nitin, S.; Deshmukh, S.G.; Perm, V. (2005): Service quality models: a review, International Journal of Quality and Reliability Management, 22, 913-949.

Robins, J.M. Y Wang, N., (2000), Inference for imputation estimators, Biometrika, 87, 113-124.

Schafer J.L. \& Graham, J. W., (2002), Missing Data: Our View Of The State Of The Art, Psychological Methods, 7, 147-177.

Snoj, B.; Mumel, D. (2002): The Measurement Of Perceived Differences In Service Quality -The Case Of Health Spas In Slovenia, Journal Of Vacation Marketing, 8, 362-379.

Soriano, D.R. (2002): Customer's expectations factors in restaurants: The situation in Spain, 
International Journal of Quality and Reliability Management, 19, 1055-1067.

United Nations Publications (2007): Imputación de Datos: Teoría y Práctica. Vol. 54 of Serie Estudios estadísticos y prospectivos, N. York.

Useche L. and Mesa D. (2006). Una Introducción A La Imputación De Valores Perdidos Terra. 22, 127-152.

Zhang, P., (2003), Multiple imputation: theory and method, International Statistical Review, 71, 581-592. 